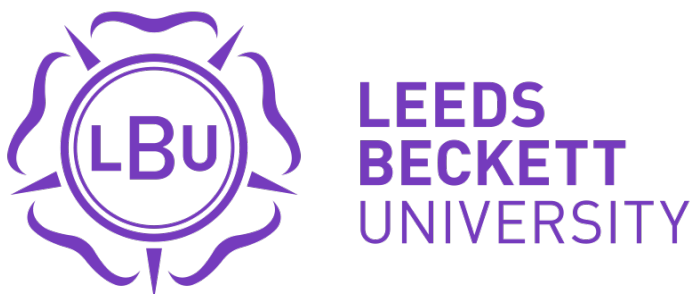

Citation:

Cardoso-Castro, P and Camacho, C (2010) La Problematica Ambiental y los Centros de Desarrolo Academico. Poliantea, 6 (11). pp. 253-270. ISSN 2145-3101 DOI: https://doi.org/10.15765/plnt.v6i11.209

Link to Leeds Beckett Repository record:

https://eprints.leedsbeckett.ac.uk/id/eprint/3532/

Document Version:

Article (Published Version)

Creative Commons: Attribution-Noncommercial-No Derivative Works 4.0

The aim of the Leeds Beckett Repository is to provide open access to our research, as required by funder policies and permitted by publishers and copyright law.

The Leeds Beckett repository holds a wide range of publications, each of which has been checked for copyright and the relevant embargo period has been applied by the Research Services team.

We operate on a standard take-down policy. If you are the author or publisher of an output and you would like it removed from the repository, please contact us and we will investigate on a case-by-case basis.

Each thesis in the repository has been cleared where necessary by the author for third party copyright. If you would like a thesis to be removed from the repository or believe there is an issue with copyright, please contact us on openaccess@leedsbeckett.ac.uk and we will investigate on a case-by-case basis. 


\title{
La problemática ambiental y los centros de desarrollo académico
}

\author{
Fecha de recepción: 6 de septiembre de 2010 - Aprobación: 11 de octubre de 2010
}

\section{Clemencia Camacho Delgado, Pedro Pablo Cardoso}

\section{Resumen}

Este artículo se presenta como una segunda parte del ya publicado en el número 10 de Poliantea "Revisión del problema ambiental y su gestión". Aquí se hace una revisión específica de la gestión ambiental llevada a cabo por las universidades desde sus inicios en 1950, de los compromisos adquiridos y las asociaciones que se fueron consolidando a través del tiempo.

El recorrido muestra concepciones y hechos de la cultura ambiental en las diferentes instituciones de educación superior, su interés en los procesos de formación en el tema y responsabilidad social como centros de generación de soluciones a los problemas de deterioro ambiental global. Finalmente se incluye un cuadro secuencial de las iniciativas y eventos realizados por universidades desde los años 1950. Se busca mostrar la importancia de las acciones y realizaciones de las diversas comunidades universitarias en el tema ambiental

\section{Abstract}

This article is presented as the second part of the one published on Issue 10 of Poliantea: Environmental
Review and Management Problem. Here, we review specific environmental management undertaken by the universities since its inception in 1950, along with its commitments and partnerships that were consolidated over time. The study shows conceptions and events of environmental culture in the different institutions of higher education, its interest in the processes of training in the subject and social responsibility as centers of generation of solutions to the problems of global environmental degradation. Finally, it includes a picture sequence of initiatives and events organized by universities since the fifties. It aims to show the importance of the various actions and accomplishments of the different university communities regarding environmental issues.

\section{Palabras clave}

Medio ambiente, gestión ambiental, instituciones educativas.

\section{Key Words}

Environment, environmental management, educational institutions

\section{Gestión ambiental en las universidades}

Una de las primeras acciones universitarias de alto impacto en el desarrollo de actividades para el logro de la sostenibilidad fue la Asociación Internacional de Universidades
(IAU, por su sigla en inglés), fundada en 1950 como parte del programa Unesco para la conformación de la Asociación Mundial de Instituciones de Educación Superior, que reúne organizaciones e instituciones 
de más de 150 países, para la reflexión y la generación de actividades en temas comunes a los participantes y colaboradores, y cuenta con el apoyo de organizaciones de carácter internacional, regional y local relacionados con la educación superior. Presta sus servicios de manera prioritaria a las organizaciones afiliadas, así como a organizaciones, instituciones y autoridades no afiliadas relacionadas con la educación superior; incluye también a individuos con capacidad de decisión sobre políticas educativas, especialistas, administradores, profesores, investigadores y estudiantes.

El propósito de esta asociación es facilitar el desarrollo del cumplimiento de las obligaciones propias de los centros de

\section{Reseñas de autores \\ Clemencia Camacho Delgado \\ Politécnico Grancolombiano \\ ccamacho@poligran.edu.co \\ Bióloga y MSc. de la Pontificia Universidad Javeriana, con especialización en Gestión Ambiental de la Universidad Externado de Colombia. Diplomada en Docencia Universitaria, Enfoques Pedagógicos Contemporáneos, Investigación, y en Estudios de China Contemporánea. Cuenta con varios artículos publicados en revistas uni- versitarias. Directora de Investigación del Politécnicos Grancolombiano y del Grupo en Educación e Investigación de la institución. Líder investigadora de la Comunidad Latinoamericana Urdimbre Educación e Investigación.}

\section{Pedro Pablo Cardoso}

Business School de la Universidad de Hull ppccardoso2@gmail.com

Biólogo Marino de la Universidad Jorge Tadeo Lozano con Máster en Planeación y Auditoria Ambiental Empresarial del Instituto de Investigaciones Ecológicas, Málaga (España) y Máster en Comercio Internacional del Instituto de Estudios Bursátiles, Universidad Complutense - Bolsa de Madrid. Durante los últimos diez años ha combinado su actividad académica como catedrático-investigador en diferentes programas de pre y posgrado, con sus actividades de emprendimiento y consultoría en sistemas integrados de gestion (ISO 9000-14000), negocios internacionales y marketing. En la actualidad cursa su tercer año de estudios de doctorado en la Business School de la Universidad de Hull (Inglaterra). educación superior, que en su calidad de instituciones sociales deben promover, por medio de la enseñanza, la investigación y la prestación de servicios, los principios de libertad, justicia, dignidad humana y solidaridad; contribuyendo, por medio de la cooperación internacional, al desarrollo y la creación de formas de asistencia moral y material para el fortalecimiento de la educación superior en general (IAU, 1993).

Para ello, establecen como principios fundamentales que las universidades deben seguir los siguientes parámetros:

- El derecho a buscar el conocimiento para su propio bien, y el de seguir el camino, dondequiera que la búsqueda de la verdad pueda conducir

- La tolerancia a las opiniones divergentes y libertad ante la interferencia política.

Esta asociación de universidades luego participaría en el desarrollo de la gestión ambiental universitaria en varias ocasiones, como se señala más adelante.

En 1959 se realizó la conferencia de rectores, presidentes y vicerrectores de universidades europeas. Esta reunión dio inicio, en 1973, al Comité de Rectores de Estados Miembros de la Comunidad Europea (que en 1989 simplificaron su nombre a Confederación de Rectores de la Unión Europea, y sería conocida e instituida como CRE en 1996 con el nombre de Asociación de Universidades Europeas), con influencia en la definición de políticas de gestión ambiental y desarrollo sostenible universitario.

Entre sus parámetros y términos de gestión influye en la creación de marcos regulatorios europeos, por medio de la educación superior y la investigación. Este es uno de los grupos y programas universitarios más efectivos cuya evolución se pre- 
senta en los documentos de "Tendencias" publicados por la EUA European University Association (Sursock y Smidt, 2010), y que junto con otras iniciativas universitarias surgidas y desarrolladas en forma paralela se describen con más detalle en este texto.

En 1969 surgió "El Tercer Mundo primero" (Third World First), un grupo de estudiantes y en general de acción social, dedicado a recolectar dinero para ayudar a las naciones pobres del planeta. Esta preocupación, con el paso del tiempo, se transformó en "La gente y el planeta" (People and Planet 2004), que desarrollaría desde algunas universidades europeas, principalmente del Reino Unido, programas de mejoramiento y educación ambiental en más de sesenta instituciones universitarias por intermedio de su programa Going Green.

Como continuación de las reuniones de universidades europeas iniciadas en 1959 (Carta Magna de las Universidades Europeas-Magna Carta Universitarium, Declaración de Bolonia), se dio la Reunión de Universidades Europeas, gestada en 1986 y realizada en 1988 con motivo de los novecientos años de la Universidad de Bolonia. El documento consiste en una declaración firmada por las universidades más antiguas del Viejo Continente, en el que se expresan los cuatro principios fundamentales del quehacer universitario para el futuro (de Europa), entre los cuales se hace mención a conceptos de desarrollo sostenible y a la relación entre desarrollo económico y bienestar ambiental para las generaciones futuras (Universidad de Bolonia, EUA, 1987).

\section{Programas ambientales universitarios}

Entre estos programas de mejoramiento ambiental universitario se destaca el pro- grama Oikos (1997), creado en 1987 por iniciativa de un grupo de estudiantes en St. Gallen (Suiza), que inicialmente pretendía incluir conferencias y sesiones de trabajo sobre asuntos ambientales en el programa de estudios de las ciencias económicas y administrativas. En 1989 Oikos creó la Conferencia Gerencia Ecológica y se convirtió en una asociación con más de trescientas empresas asociadas, generando así un proceso de reingeniería en la industria Suiza acorde con los principios de sostenibilidad.

En 1988 se creó el Cooperation Programme in Europe for Research on Nature and Industry through Coordinated University Studies (Copernicus), como órgano filial de una red de universidades constituida por el CRE (Copernicus Campus, 2007).

En 1990 se llevó a cabo la Reunión de Presidentes de Universidades por un Futuro Sostenible, en la que se originó la Declaración de Tallories, la cual se basó en funciones cívicas y responsabilidades sociales de la educación superior. En esta reunión se estableció que las universidades signatarias en representación de todas las regiones del mundo (diecinueve universidades firmaron la carta de compromisos), se comprometen a cumplir con diez preceptos que tienen por propósito hacer de las universidades centros de generación de soluciones a los problemas de deterioro ambiental global. En la declaración se consideran aspectos, como establecer actividades y programas educativos tendientes a fomentar el conocimiento general de la problemática ambiental, la educación para el desarrollo sostenible y educar mediante el ejemplo por medio de la adopción de 
prácticas ambientalmente favorables (ULSF, 2008; The Talloires Declaration, 1990.

En 1990 se creó la Fundación Oikos (Oikos, 1990), centro independiente, pero relacionado con el sector académico, cuyos objetivos son la integración de las dimensiones ecológica y social mediante la enseñanza de la economía, la administración

Ética ambiental: las universidades deberán promover entre sus profesores, estudiantes ypoblación relacionada, patrones de consumo sostenible y estilos de vida ecológicos, al tiempo que ponen en marcha programas de formación tendientes a desarrollar competencias útiles para la enseñanza de estos aspectos $y$ se crea bibliografía especializada. de negocios, y la ejecución de proyectos de investigación en el campo del desarrollo sostenible, economía y gerencia sostenible. Labores que ejecuta mediante la financiación de proyectos de investigación universitarios.

De este modo la asociación se establece como una plataforma neutral de estudio, discusión y desarrollo de los contenidos del que luego sería conocido como capítulo 36 de la Agenda 21 (Declaración de Río 1992, sobre medio ambiente y desarrollo). Al reconocer que se requería de nuevos conceptos y valores sociales para lo cual la educación, en particular la universitaria tendría una función crítica como lo es la de formar a los futuros maestros y tomadores de decisiones, que en suma mejorarían la capacidad de la población global para abordar los actuales y futuros problemas ambientales y generar respuestas de carácter sostenible, desarrollando el sentido de ética y responsabilidad social.

Se estableció que una de las responsabilidades de las universidades es la formación de profesionales necesarios con competencias en investigación, tecnología, ciencias naturales, humanas y sociales, capaces de promover la práctica de la ética ambiental en la sociedad de acuerdo con lo estipulado en la carta magna de las universidades europeas y las recomendaciones de la Unced (1992). Para ello las universidades son inducidas a implementar por medio de las actividades de Oikos los siguientes principios de acción:

- Compromiso institucional: las universidades deberán demostrar compromiso real con el cumplimiento de las prácticas de protección ambiental y desarrollo sostenible en su gestión académica.

- Ética ambiental: las universidades deberán promover entre sus profesores, estudiantes y población relacionada, patrones de consumo sostenible y estilos de vida ecológicos, al tiempo que ponen en marcha programas de formación tendientes a desarrollar competencias útiles para la enseñanza de estos aspectos y se crea bibliografía especializada.

- Educación para empleados universitarios: las universidades deberán proveer a sus empleados educación, entrenamiento y apoderamiento relativo a asuntos ambientales de modo que aquellos puedan aplicar dichos conocimientos en el desarrollo de sus actividades de forma responsable

- Programas de educación ambiental: las universidades deberán incorporar una perspectiva ambiental a su trabajo y crear programas de educación ambiental involucrando a profesores, investigadores y estudiantes.

- Interdisciplinaridad: las universidades deberán promover la interdisciplinaridad, la educación colaborativa $y$ los programas de investigación relacionados con el desarrollo sostenible como parte de la misión central de la institución y buscar hacer más competitivas las distintas disciplinas $y$ departamentos. 
- Diseminación del conocimiento: las universidades deberán apoyar esfuerzos tendientes a saldar las deficiencias de información de estudiantes, profesionales, directivos, y público en general, mediante la preparación de material didáctico, promoción de centros de lectura especializados y establecer programas de entrenamiento.

- Red de trabajo: las universidades deberán promover el trabajo en redes interdisciplinarias de expertos ambientales locales, nacionales, regionales e internacionales, con el propósito de colaborar y participar en proyectos ambientales comunes en las áreas de educación e investigación. Para ello se deberá estimular la movilidad de estudiantes y profesores.

- Alianzas: con el ánimo de diseñar e implementar estrategias y planes de acción coordinados, las universidades deberán tomar la iniciativa en la creación de alianzas con otros sectores de la sociedad.

- Programas de educación continuada: las universidades deberán crear programas de educación especializados para diferentes grupos de interés como negocios, gobierno, ONG y medios de comunicación, entre otros.

- Transferencia de tecnología: las universidades deben contribuir a programas de ecuación diseñados para facilitar la transferencia tecnológica, la innovación y los métodos de gestión avanzados.

\section{Asociaciones e institutos ambientales de universidades}

En 1990 surgió la iniciativa de creación de la Asociación de Universidades Líderes por un Futuro Sostenible (ULSF, 2008, por su sigla en inglés), como una organización internacional sin ánimo de lucro que aúna los esfuerzos de colegios y universidades para hacer del desarrollo sostenible un tema principal en los programas de educación, investigación, servicio de extensión y operaciones de los centros educativos. Y se constituye como agencia del secretariado de las universidades signatarias de la declaración de Talloires.

En 1991 apareció en escena la Asociación Internacional de Universidades (IAU) al definir como proyecto prioritario el desarrollo sostenible y con participación en la Conferencia de Halifax (1991), con lo que daría inicio a una serie de intervenciones en múltiples eventos internacionales relacionados con la función de los centros de educación superior y la participación universitaria en el logro de los propósitos del desarrollo sostenible. Entre algunas de esas intervenciones figuran: la Mesa Redonda para el Seguimiento de los Acuerdos de Río (Declaración de Kyoto, 1993); la creación en 1997 de un grupo de trajo para la educación en desarrollo sostenible, derivado de los resultados del plan Década para la Erradicación de la Pobreza 1996-2006 de la ONU; preparación de un plan de trabajo para la educación y el desarrollo humano sostenible, en la reunión de la Unesco sobre educación superior (1998); creación de la Asociación Global de Educación Superior para la Sostenibilidad (GHESP, 2000,), entre otras.

En 1992 se oficializó la constitución de la ULSF, que aplicó programas de investigación, gestión de recursos, apoyo en la medición de desempeño en sostenibilidad de las universidades afiliadas, y desarrollo 
de alianzas entre universidades internacionales para el avance en la educación sobre temas de desarrollo sostenible.

Este programa define el desarrollo sostenible como una práctica, que implica una actitud crítica frente a los movimientos "verdes" por parte de los centros de educación superior, que ofrecen soluciones justas y económicamente viables tendientes a asegurar recursos suficientes para satisfacer las necesidades de las generaciones futuras. Un verdadero centro de educación superior sostenible debe entonces hacer énfasis en sus programas académicos y de investigación, el desarrollo de temas relacionados con el logro de estos propósitos, preparando estudiantes para que contribuyan como futuros profesionales a la conformación de una sociedad más justa y ambientalmente responsable. Para ello, los centros educativos deben funcionar como una comunidad sostenible, fomentando el consumo sostenible de comida y energía, tratando a sus diversos miembros con respeto y promoviendo estos mismos valores en sus comunidades vecinas.

En 1993 se fundó el Instituto para la Economía y el Medio Ambiente (EEI, Janssen, 2000), en la Universidad de St. Gallen, un departamento especializado e independiente de la fundación Oikos. Ese mismo año se firmó la Carta Universitaria Copernicus por el Desarrollo Sostenible (Michelsen, 2003), como respuesta a los compromisos planteados en la cumbre de Río 92, que dejó profundas huellas en la conciencia ambiental de las universidades europeas y su compromiso con la preservación y prestación de garantías para el futuro de la humanidad. Además, hace llamado a los gobiernos, la administración pública, la comunidad científica, las universidades, las organizaciones no gubernamentales y las organizaciones globales para, que den prioridad a la educación y trabajen en el fortalecimiento de los sistemas educativos para enseñar sobre el desarrollo sostenible.

En la actualidad más de 320 universidades e instituciones de educación superior de 38 países europeos han firmado la carta que declara se dará al desarrollo sostenible un lugar importante en sus actividades., lo cual implica que los currículos, la gestión institucional, y sus demás servicios a la sociedad local y regional responderán a un balance responsable entre los aspectos económicos, ecológicos y socioculturales.

En 1994, la Universidad de la ONU creó el programa Zero Emissions Research Initiative (ZERI), cuando este centro estaba bajo la dirección de su tercer rector, Gurgulino de Souza y con el liderazgo de Gunter Pauli. La iniciativa de investigación se concentró en la creación de sistemas industriales que replicaran el funcionamiento de los ciclos sostenibles naturales que no producen desechos. Este programa generó iniciativas coma las de universidades cero emisiones, y más adelante, investigaciones para la creación de sistemas de producción energética de cero emisiones.

En 1995, y con el ánimo de contribuir con el logro de los compromisos derivados de la Agenda 21, en particular los relacionados con la necesidad de generar conciencia y conocimiento sobre la problemática ambiental, así como instrumentos de gestión para la solución de la misma, entre ellos los de transferencia de tecnología, mecanismos de cooperación e incremento de la capacidad de gestión, fue creada la Organización de Universidades por el Desarrollo Sostenible y el Medio Ambiente (OIUDSMA), una red de 
universidades que se formalizó en una primera reunión celebrada en Costa Rica, y que contó en su constitución con la presencia de representantes de veinte universidades de Iberoamérica (OUIDSMA, 1995).

En 1996 el ZERI fue fusionado a un nuevo instituto de estudios avanzados (Institute of Advanced Studies, UNU-IAS). El director de este nuevo instituto, Della Senda, reconoció el potencial del concepto cero emisiones en sistemas industriales y sus implicaciones para la generación de cambios sociales en la configuración de comunidades sostenibles. En consecuencia se inicia el proceso de diseño de un sistema de gestión ambiental (SGA) universitario para el campus de la sede principal de la universidad en Tokio, que luego fue certificado con la norma IsO 14000 (UNU-IAS, 1994).

Este mismo año fue creada la Asociación Ambiental de Universidades y Colegios (EAUC, 1996), cuyo propósito fue el de elevar el desempeño ambiental de las instituciones educativas asociadas y los estándares, mediante mejorando el nivel de gestión ambiental de las mismas. Propósito que fue inicialmente logrado mediante:

- Creación de un foro en donde se compartieron experiencias e información entre representantes de diferentes colegios y universidades.

- Divulgación de las mejores prácticas ambientales, las estrategias para el reverdecimiento del campus y las estrategias para el reverdecimiento de programas académicos

Esta asociación además se seguir vigente tiene nuevos proyectos:

- Ecocampus

- Universidades que participan activamente (Universities that count)
- Programa de sostenibilidad de campus (Campus sustainability program)

- Compromiso de universidades e institutos de educación superior con el cambio climático. (Universities and colleges climate commitment)

Esta asociación rápidamente desarrolló un portafolio de servicios para el mejoramiento del desempeño ambiental de las instituciones afiliadas, las cuales en un período muy corto incrementaron su número. El propósito de esta prestación de servicios es garantizar el acceso de las instituciones miembros a la información sobre la legislación ambiental, las tecnologías para el mejoramiento del desempeño ambiental aplicables a centros educativos, mejores prácticas e investigación.

En 1997 Oikos decidió internacionalizar sus actividades y fortalecer su capacidad de estudios en temas relacionados con el componente social y ecológico, para lo cual ofreció su programa a otras universidades de modo que en 1998 existía ya en universidades como Colonia, Praga, Estocolmo y Viena, y con administración central de los diferentes programas de educación en St. Gallen.

En 1999 Copernicus se independizó, se constituyó como un programa independiente del CRE y se presentó el programa Copernicus-Campus 1997), orientado a promover la transferencia de conocimiento entre las universidades e identificar, promover y diseminar buenas prácticas y estrategias para hacer de la sostenibilidad un eje central de la vida universitaria, con el ánimo de contribuir al desarrollo sostenible de Europa. Así adoptó un enfoque proactivo en el cual las universidades determinan autónomamente, pero de forma coordinada cuál 
puede ser su aporte para lograr las reformas del Proceso de Bolonia de cara a la globalización y contribuir con el conocimiento europeo, mediante la activa participación en el programa de la década de la educación para el desarrollo sostenible de la ONU.

Este mismo año, la universidad de la ONU desde su sede principal, lanzó el Foro Cero Emisiones (ZEF) que cuenta con representantes del sector académico, empresarial, gubernamental y científico y que, en conjunto persiguen tres objetivos (ONU, 2001):

- Investigación: ZEF promueve en el ámbito internacional la interdisciplinariedad en investigación, en función de comprender las tendencias sociales y tecnológicas, y para ello emplea una gran variedad de metodologías como, por ejemplo, balances de flujos de materia y energía. Esto permitiría en un futro el desarrollo de escenarios para la ecoreestructuración empleando instrumentos, como el análisis del ciclo de vida para evaluar las implicaciones ambientales de nuestras tecnologías, diseños industriales y políticas micro y macroeconómico.

- Capacidad de construcción: mediante el trabajo con universidades líderes, el ZEF promueve el desarrollo de centros multidisciplinarios de excelencia en cero emisiones. El foro proveerá a los miembros de estas redes de conocimiento la oportunidad de compartir sus experiencias y saber cómo (knowhow) con gestores de políticas públicas, gerentes de grupos empresariales y líderes comunitarios.

- Redes: el ZEF colabora con el desarrollo institucional, mediante la fundación de agencias, puntos de apoyo y asesorías a gobiernos, empresarios y comunidades para estimular iniciativas locales y regionales de cero emisiones, por medio de las actividades industriales $y$ sociales en el planeta. En este contexto, ZEF desempeña un papel clave al promover la incorporación de objetivos de cero emisiones en estamentos de definición de políticas gubernamentales $\mathrm{y}$ programas de colaboración con ONG e institutos de investigación.

Como consecuencia, en el ámbito internacional ha madurado la iniciativa de universidades cero emisiones, las cuales en la actualidad concentran sus esfuerzos de investigación, principalmente en el desarrollo de mecanismos de generación de energía sin emisiones (celdas de hidrógeno) y sistemas alternativos al motor de combustión para la generación de movilidad; programas de investigación que con regularidad son cofinanciados por empresas automotrices y del sector energético.

En 2001, se constituyó la asociación para el mejoramiento del desempeño ambiental de los centros de educación superior -Higher Education Environmental Performance Improvement (Heepi, 2010)-, de acuerdo con las directrices de la iniciativa de Buenas Prácticas de Gestión del Consejo- Fundación de Educación Superior, la Asociación Ambiental de Universidades y Colegios (EAUC) y la Asociación de Directores del Reino Unido.

Este programa, cuya tercera fase será ejecutada hasta 2007, se propone mejorar el desempeño ambiental de los centros de educación superior por medio de:

a. Estimular encuentros de educación superior, la difusión de mejores prácticas 
mediante el desarrollo para ello de bases de datos, estadísticas y nuevos sistemas de indicadores ambientales.

b. Elevar la capacidad operativa del personal involucrado o con responsabilidad inmediata sobre asuntos relacionados con el desempeño ambiental de las instituciones, para alcanzar mejoras en sus respectivas instituciones, mediante seminarios, y el análisis de casos de estudio relacionados principalmente con mejores prácticas.

En 2003 el Grupo People and Planet creó el programa Going Green para los centros de educación de Inglaterra (y el Reino Unido), para lo cual desarrolló manuales y bases de datos de mejores prácticas de las universidades inscritas en el programa, así como eventos diseñados para involucrar a la población estudiantil en la gestión ambiental (People \& Planet, 2004). Entre los lineamientos del programa se sugiere a las universidades adoptar las siguientes recomendaciones:

- Apoyo de la dirección de la universidad, pues el apoyo de un vicerrector o del rector es fundamental para el éxito de un programa de gestión y mejoramiento ambiental.

- Personal de tiempo completo dedicado a la gestión ambiental. Para lograr objetivos, establecer prioridades, definir metas y realizar actividades solo es posible con personas dedicadas de tiempo completo a esta actividad.

- Completa revisión e investigación de los impactos ambiéntales de la institución. Solo por medio de la medición y valoración de los impactos ambientales se logra la identificación de las oportunidades de mejora, y esta solo será posible con programas de seguimiento y control.
- Política ambiental pública, para dar un marco formal e institucional a las intenciones de mejoramiento ambiental y ofrecer un punto de referencia a todas las actividades que en este sentido se emprendan.

En 2005, la ONU dio inicio a las actividades de su plan Década de la Educación para el Desarrollo Sostenible 2005-2014 (Unesco, 2006) proclamado en la 57 sesión de la Asamblea General de la ONU en 2002; que contempla temas claves de acción como el de la igualdad de género, la promoción de la salud, el medio ambiente, el desarrollo rural, el consumo sostenible, la diversidad cultural, la paz, la seguridad humana y la urbanización sostenible.

Para ello ha diseñado una serie de mecanismos de participación que tienen como gestor las oficinas de la Unesco, a las cuales se han adherido participantes de varias universidades mundiales (Unesco, ESD, 1995).

En los últimos años, 2007, la UsLF se independizó del Centro de Respeto de Vida y Ambiente (CRLE). En la actualidad funciona como una organización virtual y aún mantiene su posición como secretaría de los signatarios de la declaración de Tallories.

En 2008 EAUC tiene la iniciativa de crear edificios de alto rendimiento en las universidades y colegios, y tiene para 2009 un amplio programa de desarrollo profesional para el personal que trabaja en la sostenibilidad en las universidades y colegios.

La conferencia de educación para el desarrollo sostenible se realizó en 2009 en la universidad de Bournemouth. Ese mismo año se llevó a cabo el Evento Internacional de la Educación Verde, realizado por la IAU, que también gestionó la VIII Conferencia 
Mundial del Medio Ambiente en Goa, en India (IAU, 1993).

\section{Acciones ambientales latinoamericanas en el contexto universitario}

En Latinoamérica se encuentra el Centro Latinoamericano de Economía Social (Claes), fundado en 1989 con sede en Montevideo. Claes (2010) es una organización dedicada a la investigación, acción y promoción de la ecología social, a partir de la cual nace una iniciativa llamada Red latinoamericana y caribeña de ecología social (RedLaces, 2010), la cual agrupó a instituciones y a personas interesadas en la ecología social y humana, y cuyos objetivos son promover la reflexión y análisis, vinculado a una práctica, y el compromiso ético con la defensa de la vida.

Para 1985 en Bogotá, se llevó a cabo el primer Seminario sobre Universidad y Ambiente para América Latina y el Caribe. Representantes de 59 universidades e instituciones con carácter ambiental de 22 países de la región, firmaron la Carta de Bogotá sobre Universidad y Medio Ambiente, en la que se valora la dimensión ambiental en el currículo, las posibilidades de investigación y proyección social y, así mismo, se pone a discusión la necesidad de fortalecer en cantidad y calidad a los profesionales que realizarán dichas tareas (Icfes, UNEscoPNUMA, 1988).

En 1999, la Unión de Universidades de América Latina y del Caribe (Udual), hizo pública la declaración sobre la Universidad Latinoamericana en el Siglo XXI (Udual, 1999). En 2003, se incorporó a la Década de la Educación para el Desarrollo Sostenible y propuso una agenda de trabajo a sus afiliados, con el fin de comprometer a las universidades en un análisis y reflexión sobre el desarrollo sostenible y las acciones, que se deben realizar para lograrlo, incluyendo el control de sus propios impactos ambientales (Tünnermann, 2003).

Es importante el papel de la Red de formación ambiental para América Latina y el Caribe del PNUMA (1999), que está orientado hacia la construcción, sistematización y defunción de conocimientos, saberes, métodos y técnicas para la gestión ambiental, que sirvan como materiales básicos para los programas de formación ambiental y como un instrumento que apoyen las políticas de desarrollo sustentable de la región en los diferentes niveles de gobierno, así como para capacitar a los diferentes sectores sociales, tanto en los niveles profesionales como en las acciones ciudadanas y los programas de desarrollo comunitario.

En nuestro país contamos con la Red colombiana de formación ambiental (RCFA, 2006), que forma parte de la red latinoamericana. La Secretaría General está a cargo de la Oficina de Educación y Participación del Ministerio de Ambiente, Vivienda y Desarrollo Territorial (MAVDT), y la integran las instituciones que hacen parte de su junta directiva, 32 universidades y varias ONG. Esta red (RCFA) ha organizado los seminarios internacionales desde el año 2000 mostrando la importante labor de muchas universidades en la formación de sus estudiantes y gestión ambiental de sus campus (UDCA, 2005).

El IV Seminario Internacional Universidad y Ambiente (2007), organizado por la Universidad de Ciencias Aplicadas y Ambientales (UDCA) y el Politécnico Grancolombiano tuvo como tema central la "Gestión ambiental institucional y ordenamiento de campus universitarios”. Esto 
muestra el interés por un buen desempeño ambiental en las instituciones de educación superior, reconocimiento y experiencia, que es difundida entre ellas para mejorar sus procesos (Rojas, 2008).

Existen muchas propuestas para el manejo de un sistema de gestión ambiental en las diferentes universidades del país, de hecho universidades como la UDCA (Sáenz, 2007), Politécnico Grancolombiano (Camacho, 2008), Javeriana (Ideade, 2010), Nacional (IDEA, 2010), entre otras presentan sus compromisos con el SGA, por medio de su política ambiental para establecer procesos y metas claras por cumplir.

\section{Conclusiones}

Finalmente, esta revisión muestra muchas y variadas acciones de asociaciones a instituciones universitarias unidas, en la búsqueda de soluciones a los impactos ambientales generados por el desarrollo de las actividades humanas. Es indudable que por medio de la educación de ciudadanos y comunidades se busca llegar a entender que el tema ambiental es un elemento de la formación interdisciplinaria, orientado por valores hacia la solución de problemas con relevancia en lo local en una mirada global.

En consecuencia, las instituciones educativas tienen un verdadero compromiso con las acciones que desarrollan en el plano de la gestión ambiental como agentes de educación de la realidad ambiental. De esta manera estarán mostrando el reconocimiento a los retos ambientales, que exigen profundas transformaciones de valores y prácticas. Es indudable que como semillero de líderes y lugar de experimentación, la universidad contribuye con sus acciones y compromisos al desarrollo social, económico y político de su entorno. De esta forma se convierte en eje clave de su comunidad, mediante la promoción de proyectos, que apoyen el conocimiento del entorno y las acciones para favorecerlo o mejorarlo, logrando una natural relación de los equipos y de los miembros de las diversas comunidades universitarias en el tema ambiental.

Como exploración final en la tabla 1 se presenta la secuencia de las iniciativas y eventos realizados por universidades desde los años 1950.
Finalmente, esta revisión muestra muchasy variadas acciones de asociaciones a instituciones universitarias unidas, en la búsqueda de soluciones a los impactos ambientales generados por el desarrollo de las actividades humanas.

Tabla 1. Secuencia de iniciativas y eventos por universidades desde 1950

\begin{tabular}{|l|l|}
\hline \multicolumn{1}{|c|}{ Iniciativas universitarias } & \multicolumn{1}{c|}{ Eventos mundiales } \\
\hline & 2010 - EAUC Catorce Conferencia Mundial de EAUC - \\
Universidad de Bangor 2010
\end{tabular}


2008 - EAUC

Edificios de alto rendimiento en las universidades y colegios

Universidad de Sheffield

2006 UNU-Unesco Mesa de trabajo sobre el

establecimiento de una agenda estratégica de

2006 - Encuentro del GHESP

investigación para el Decenio de las Naciones Unidas de

Yokohama, Japón, agosto

la Educación para el Desarrollo Sostenible

Paris, febrero 20 a 22

2005-2006 IAU: GHESP Chair

2005 - IAU

Participates en (Halifax Consultation)

Canadá

The (report of this consultation) (rtf, $664 \mathrm{~kb}$ ) is available

online.

2005 - Reunión de Nagoya (UNU-IAS) Japón

2005-2014 - Década de las Naciones Unidas para la

Educación para el Desarrollo Sostenible (United Nations

Decade of Education for Sustainable Development)

2005-2014 - Década para la Acción de las Nacionales

Unidas para el Agua para la Vida ("Water for Life")

2005 - (CSD-13)

2004 - Main focus: Development (GHESP RP) and IAU

2004 - (CSD-12)

2003 - La IAU lleva a cabo la Conferencia Internacional sobre Educación para un Futuro Sostenible: que da forma al papel de los centros de educación superior para el desarrollo sostenible.

("Education for a sustainable future: Shaping the practical role of higher education for sustainable development") Universidad de Charles

Praga, República de Checoeslovaquia.

2003 - People \& Planet crea el programa Going Green

2002 - GHESP firma un nuevo memorando de acuerdo (Memorandum of agreement) y se reafirma por cinco años como miembro clase II de la Cumbre de Johannesburgo.

Con el propósito de movilizar universidades y centros de educación superior al apoyo de las premisas del desarrollo sostenible, un plan de acción renovado (Renewed Action Plan) es desarrollado entre la IAU, ULSF y UNESCO.

2001 - GHESP adopta la (Lüneburg Declaration on Higher Education) 2001 - Creación del Heepi

2003-2012 - Década de las Naciones Unidas para la Erradicación del Analfabetismo

(United Nations Literacy Decade)

2003 - (CSD-11) En su primer encuentro desde la cumbre de Johannesburgo, se hace un programa y un plan de trabajo para los próximos quince años.

2002 - El Encuentro Mundial para el Desarrollo Sostenible emite la declaración de desarrollo sostenible de Johannesburgo

(Johannesburg Declaration on Sustainable Development) 2002 - Plan de implementación de Johannesburgo. (Johannesburg Plan of Implementation) 2002 - (CSD-10) Preparación para el Encuentro Mundial de Desarrollo Sostenible

2001-2010 - (International Decade for a Culture of Peace and Non-violence for the Children of the World) 2001 - (CSD-9) Enfoque en energía para el desarrollo sostenible, atmósfera, transporte, información para la toma de decisiones y mecanismos de participación. Cooperación internacional para un mejor ambiente y preparación deMmundial de Desarrollo Sostenible. 


\begin{tabular}{|c|c|}
\hline $\begin{array}{l}2000 \text { - En respuesta a lo establecido en el capítulo } 31 \\
\text { de la Agenda 21, se establece la Alianza de centros de } \\
\text { educación superior para el desarrollo sostenible } \\
\text { (Global Higher Education for Sustainability Partnership } \\
\text { (GHESP)) }\end{array}$ & $\begin{array}{l}2000 \text { - (Dakar Framework for Action) } \\
\text { Educación para todos: logrando nuestros compromisos } \\
\text { colectivos } \\
2000 \text { - Carta de la Tierra } \\
\text { (Earth Charter) } \\
2000 \text { - Declaración del Milenio } \\
\text { (Millennium Declaration) } \\
2000 \text { - Metas de Desarrollo del Milenio } \\
\text { (Millennium Development Goals) } \\
2000 \text { - (CSD-8) Enfoque en agricultura, finanzas, manejo } \\
\text { de suelos y comercio }\end{array}$ \\
\hline & $\begin{array}{l}1999 \text { - (CSD-7) Conferencia enfocada al turismo sostenible, } \\
\text { océanos y mares, desarrollo de pequeños Estados } \\
\text { insulares, y patrones de producción y consumo }\end{array}$ \\
\hline $\begin{array}{l}1998 \text { - IAU - Realización del debate temático: } \\
\text { "Preparación para un futuro sostenible: educación } \\
\text { superior y desarrollo humano sostenible"." ("Preparing for } \\
\text { a Sustainable Future: Higher Education and Sustainable } \\
\text { Human Development"). } \\
1998 \text { - Creación del plan (Copernicus-Campus) } \\
1988 \text { - Creación del ZEF }\end{array}$ & $\begin{array}{l}1998 \text { - (Unesco World Conference on Higher Education) } \\
1998 \text { - (CSD-6) Ejecución del plan de trabajo en } \\
\text { seguimiento a la implementación de la Agenda } 21\end{array}$ \\
\hline $\begin{array}{l}1997 \text { - IAU Creación del grupo de trabajo en Enseñanza e } \\
\text { Investigación en Desarrollo Sostenible } \\
1977 \text { - Internacionalización de las actividades de Oikos }\end{array}$ & $\begin{array}{l}\text { 1997-2006 - Primera década de las Naciones Unidas para } \\
\text { la erradicación de la pobreza } \\
\text { (First United Nations Decade for the Eradication of } \\
\text { Poverty) } \\
1997 \text { - Primera declaración de las Naciones Unidas sobre } \\
\text { la responsabilidad de las generaciones presentas para con } \\
\text { las generaciones futuras } \\
\text { (First United Nations Declaration on the Responsibilities } \\
\text { of Present Generations Towards Future Generations) } \\
1997 \text { - (CSD-5) }\end{array}$ \\
\hline 1996 - Creación de la EAUC & 1996 - (CSD-4) \\
\hline $\begin{array}{l}1995 \text { - La IAU firma un Memorando de Entendimiento IISD } \\
1995 \text { - Creación de la OIUDSMA }\end{array}$ & 1995 - (CSD-3) Commission on Sustainable Development \\
\hline 1994 - Creación del ZERI & $\begin{array}{l}1994 \text { - Segunda sesión de la Comisión de las Naciones } \\
\text { Unidas para el Desarrollo Sostenible (CSD-2) realizada en } \\
\text { Nueva York, mayo } 16 \text { a } 27 .\end{array}$ \\
\hline
\end{tabular}




\begin{tabular}{|c|c|}
\hline Iniciativas universitarias & Eventos mundiales \\
\hline $\begin{array}{l}1993 \text { - Mesa redonda de la IAU en Kyoto, como } \\
\text { seguimiento a la Conferencia de Río, que adopta la } \\
\text { Declaración de Kyoto - (Kyoto Declaration) - y la Agenda } \\
\text { IAU para el Desarrollo Sostenible - (IAU Agenda } 2000 \text { for } \\
\text { Sustainable Development) } \\
1993 \text { - Declaración de St. Gallen } \\
1993 \text { - Fundación del Instituto para la Economía y el } \\
\text { Medio Ambiente IEE } \\
1993 \text { - Carta de University-Copernicus }\end{array}$ & $\begin{array}{l}1993 \text { - Primera sesión de la Comisión de las Naciones } \\
\text { Unidas para el Desarrollo Sostenible (CSD-1), realizada en } \\
\text { Nueva York, junio } 14 \text { a } 25 .\end{array}$ \\
\hline 1992 \}- Creación de la ULSF - (ULSF) & $\begin{array}{l}1992 \text { - Primer encuentro mundial de la tierra (Earth } \\
\text { Summit) para tratar problemas relacionados con la } \\
\text { protección ambiental y desarrollo socioeconómico en Río } \\
\text { de Janeiro, Brasil. } \\
\text { Una Comisión para el Desarrollo Sostenible (Commission } \\
\text { on Sustainable Development) (CSD) fue creada para } \\
\text { monitorear e implementar los acuerdos logrados en este } \\
\text { encuentro mundial. } \\
\text { Se firman la Convención para el Cambio Climático } \\
\text { (Convention on Climate Change) y la Convención para la } \\
\text { Biodiversidad (Convention on Biological Diversity). } \\
\text { Los participantes también firman la Declaración de Río } \\
\text { (Rio Declaration) y la de Principios Forestales, (Forest } \\
\text { Principles), así como la adopción de la (Agenda 21), un } \\
\text { plan de } 300 \text { páginas para el logro del desarrollo sostenible } \\
\text { en el siglo XXI. El capítulo } 36 \text { Chapter } 36 \text { promueve la } \\
\text { comunicación, la educación y el entrenamiento en asuntos } \\
\text { ambientales. }\end{array}$ \\
\hline $\begin{array}{l}1990 \text { - } 1991 \text { Conferencia y declaración de Halifax } \\
\text { (Halifax Declaration) } \\
\text { Dalhousie University, Halifax, Canadá Participación de } \\
\text { la IAU. } \\
1990 \text { - Declaración de Tallories } \\
1990 \text { - Declaración de principios Oikos } \\
1990 \text { - Propuesta de creación de la ULSF }\end{array}$ & \\
\hline $\begin{array}{l}1988 \text { - Declaración de Bolonia } \\
1988 \text { - Creación del programa Copernicus }\end{array}$ & \\
\hline 1987 - Creación de Oikos & $\begin{array}{l}1987 \text { - Definición del concepto de "desarrollo sostenible" } \\
\text { por la Comisión Bruntand (copia del Reporte Bruntland). }\end{array}$ \\
\hline & $\begin{array}{l}1972 \text { - (United Nations Conference on the Human } \\
\text { Environment) } \\
\text { Estocolmo, Suecia }\end{array}$ \\
\hline 1969 - Creación del programa Third Word First & \\
\hline $\begin{array}{l}1959 \text { - Creación del Concejo de Rectores de Estados } \\
\text { Miembros de la Comunidad Europea }\end{array}$ & \\
\hline $\begin{array}{l}1950 \text { - Creación de la Asociación Internacional de } \\
\text { Universidades - UnESCO }\end{array}$ & \\
\hline
\end{tabular}




\section{Bibliografía}

1. Camacho Delgado, Clemencia. (2008). Desarrollo sostenible: un tema de responsabilidad social universitaria. Compromiso del Politécnico Grancolombiano. En Responsabilidad social de las universidades. Tomo II. Red latinoamericana de cooperación universitaria. Buenos Aires.

2. Centro Latinoamericano de Ecologia Social (Claes) (2010) [en línea] Disponible en: www.ambiental.net/claes/ (consulta: 16 de agosto de 2009).

3. Conferencia y Declaración de Halifax. (1990-1991). Dalhousie University, Halifax (Canadá). Participación de la IAU. [en línea] En Halifax Declaration. Disponible en: http://upcommons.upc.edu/revistes/bitstream/2099/7923/1/RESUMEN_5. pdf (consulta: 16 de agosto de 2010).

4. Copernicus Campus. (2007). Copernicus-Guidelines for Sustainable Development in the European Higher Education Area. En Ccopernicus. [en línea]. Disponible en: www.dcsf.gov.uk/londonbologna/uploads/documents/COPERNICUSGuidelines. pdf (consulta: 1 de junio de 2009).

5. Declaración de Kyoto y Agenda IAU para el Desarrollo Sostenible. (1993). En IAU Agenda 2000 for Sustainable Development. [en línea] Disponible en; http://unesdoc. unesco.org/images/0011/001163/116345s.pdf (consulta: 16 de agosto de 2010).

6. European University Association (EAUC). (1996). The Enviromental Association of Universities and Collleges. Who we are? En EAUC [en línea] Disponible en: http://www.eauc.org.uk/about_us (consulta: 18 de junio de 2009).

7. Organización de las Naciones Unidas para la Educación, la Ciencia y la Cultura - Education for Sustainable Development. (Unesco, EsD). (1995) En Unesco [en línea] Disponible en: http://portal.unesco.org/education/en/ev.php-URL_

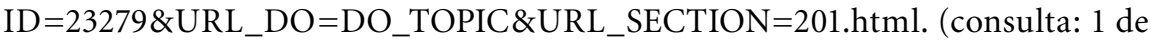
junio de 2009).

8. Global Higher Education for Sustainability Partnership (GHESP). (2000). [En línea] Disponible en: http://webapps01.un.org/dsd/partnerships/public/ partnerships/71.html (consulta: 16 de agosto de 2010).

9. Higher Education Environmental Performance Improvement (HeEPI). (2010). En HeEPI [en línea] Disponible en: http://www.heepi.org.uk/ (consulta: 16 de junio de 2010).

10. Instituto Colombiano para la Educación Superior, Organización de las Naciones Unidas para la Educación, la Ciencia y la Cultura y Programa de las Naciones Unidas para el Medio Ambiente (Ices, Unesco y PNUmA) (1988). Universidad y Medio Ambiente en América Latina y el Caribe. Seminario de Bogotá. Bogotá: Icfes y Udual, 28 octubre a 1 noviembre de 1985.

11. Instituto de Estudios Ambientales (IDEA) (2010). [en línea) Disponible en: http:// www.idea.palmira.unal.edu.co/paginas/sga/sga_index.html (consultado: 16 de agosto de 2010).

12. Instituto de Estudios Ambientales para el Desarrollo (Ideade) (2010). Sistema de gestión ambiental para el campus (SGA). [en línea] Disponible en: http://www. javeriana.edu.co/fear/ins_amb/sistema.htm (consulta: 16 de agosto de 2010).

13. International Association of Universities (IAU). (1993). International Association of Universities. Sustainable Development. Introduction. En IAU. [en línea] Disponible en: http. (consulta: 6 de julio de 2010). 
14. International Decade for a Culture of Peace and Non-violence for the Children of the World (2001-2010). Disponible en: http://www.unac.org/peacecp/decade/ background.html

15. Janssen, J. (1993). Instituto para Economía y Medio Ambiente en la Universidad de St. Gallen (IWO-HsG) (Suiza).

16. Main focus: Development GHESP RP and IAU WebPages on HESD (2004). Disponible en: http://portal.unesco.org/education/en/files/37578/11037253463GHESP_ Action_Plan_December_04.pdf/GHESP_Action_Plan_December_04. pdf[[Consultado el 16 de Agosto de 2010]

17. Metas de desarrollo del milenio (2000). Millennium Development Goals

18. Michelsen, G. (2003). Las universidades y la Agenda 21: un ejemplo de la Universidad de Lûneburg. Polis. Revista Académica Universidad Bolivariana. 1 (5) (Chile).

19. OІкоS (1997) Internacionalización de las actividades. Disponible en: http://rcci. net/globalizacion/2000/fg146.htm [ Consultado el 16 de agosto de 2010]

20. OIKOS (1997). Project portfolio a summary of main projects of Oikos International OIKOS Foundation February 2007. Disponible en: http://prague.oikosinternational. org/uploads/media/oikos_summary_of_projects.pdf [Consultado el 16 de Junio del 2010].

21. Oıкоs. (1990). OıкоS Foundation. En Oikos International [en línea] Disponible en: http://www.oikos-international.org/aboutoikos/organisation/oikosfoundation.html (consulta: 1 de junio de 2009).

22. Organización de Universidades por el Desarrollo Sostenible y el Medio Ambiente (OIUdsma) (1995) Creación. [en línea] Disponible en: http:// www.oiudsma.org/page.php?pageid=compromisos (consulta: 16 de agosto de 2010).

23. Organización de las Naciones Unidas (ONU) (2001). Informe del Consejo de la Universidad de las Naciones Unidas. Enero a diciembre de 2001. Asamblea General. Documentos Oficiales Quincuagésimo séptimo período de Sesiones Suplemento (31).

24. People \& Planet (P\&P) (2004). Going Green Report - Executive Summary. En P\&P [En línea]. P\&P. Disponible en: http://peopleandplanet.org/gogreen/report.php (consulta: 27 de octubre de 2009).

25. Programa de las Naciones Unidas para el Medio Ambiente (PNUMA) (1999). Universidad y Medio Ambiente. Formación ambiental. Órgano informativo de la Red de formación ambiental para América Latina y el Caribe, 11 (25), juliodiciembre.

26. Red colombiana de formación ambiental (RCFA) (2006). En RCFA [en línea] Disponible en: http://redcolombianafa.org/sobre_la_red.shtml (consulta: 16 de agosto de 2009).

27. Red latinoamericana y caribeña de ecología social (RedLaces) (2010). En RedLaces [en línea] Disponible en: http://www.ecologiasocial.com/ (consulta: 16 de agosto de 2009).

28. Rojas, L. (2008). Sistema de gestión ambiental (SGA). Documentos de integración para el desarrollo. Dirección Nacional de Planeación y Universidad Cooperativa de Colombia, (12), mayo. 
29. Sáenz, O. (2007). Gestión ambiental y ordenamiento de campus universitarios. Análisis y reflexiones a partir de algunas experiencias relevantes. Primer curso internacional universidad y ambiente. Gestión ambiental institucional, ordenamiento de campus universitarios. Bogotá: UDCA, UdUAL, 51 p.

30. Sursock, A.; Smidt, H. (2010). Trends 2010: a decade of change in European Higher Education. Documentos de la Asociación de la Universidad Europea (EUA). EUA Publications.

31. The Tallories Declaration. (1990). The Talloires Declaration: University Presidents for a Sustainable Future [en línea] Disponible en: http://www. iisd.org/educate/declarat/talloire.htm (consulta: 4 de junio de 2009).

32. Tünnermann, C. (2003) La universidad latinoamericana ante los retos del siglo XXI Colección Udual. Unión de Universidades de América Latina, AC Circuito Norponiente S/N Ciudad Universitaria, México, DF, marzo.

33. Universidad de Ciencias Aplicadas y Ambientales (UDCA). (2005). Memorias del Tercer Seminario Internacional Universidad y Ambiente. Bogotá: UDCA, del 8 al 10 de noviembre.

34. Unión de Universidades de América Latina y del Caribe (UduAL) (1999). En Declaración de la Universidad Latinoamericana en el siglo XXI. Unión de Universidades de América Latina (México) [en línea] Disponible en: http://www. udual.org (consulta: 4 de junio de 2009).

35. Organización de las Naciones Unidas para la Educación, la Ciencia y la Cultura (Unesco) (2006). Decenio de las Naciones Unidas de la Educación con miras al Desarrollo Sostenible (2005-2014): plan de aplicación internacional. Publicado en los Talleres de la UnEsco.

36. United Nations Conference on Environment and Development.(UNCED) (1992). En Unced [en línea] Disponible en: http://www.ciesin.columbia.edu/TG/PI/ TREATY/unced.html (consulta: 1 de junio de 2009).

37. University Leaders for a Sustainable Future (UlSF). (2008). About UlSF. En UlSF. [en línea]. Disponible en: http://www.ulsf.org/about.html. (consulta: 1 de junio de 2009).

38. Institute of Advanced Studies (UNU-IAS) (1994). UnU/Zero Emitions Research Initiative. En UNU [en línea]. Disponible en: http://www.ias.unu.edu/sub_page. aspx? catID $=5 \&$ ddlID $=468$ (consulta: 1 de junio de 2009). 
270

OLIANTEA 


\section{Suplemento Especial}

\section{Corporación Escenarios}

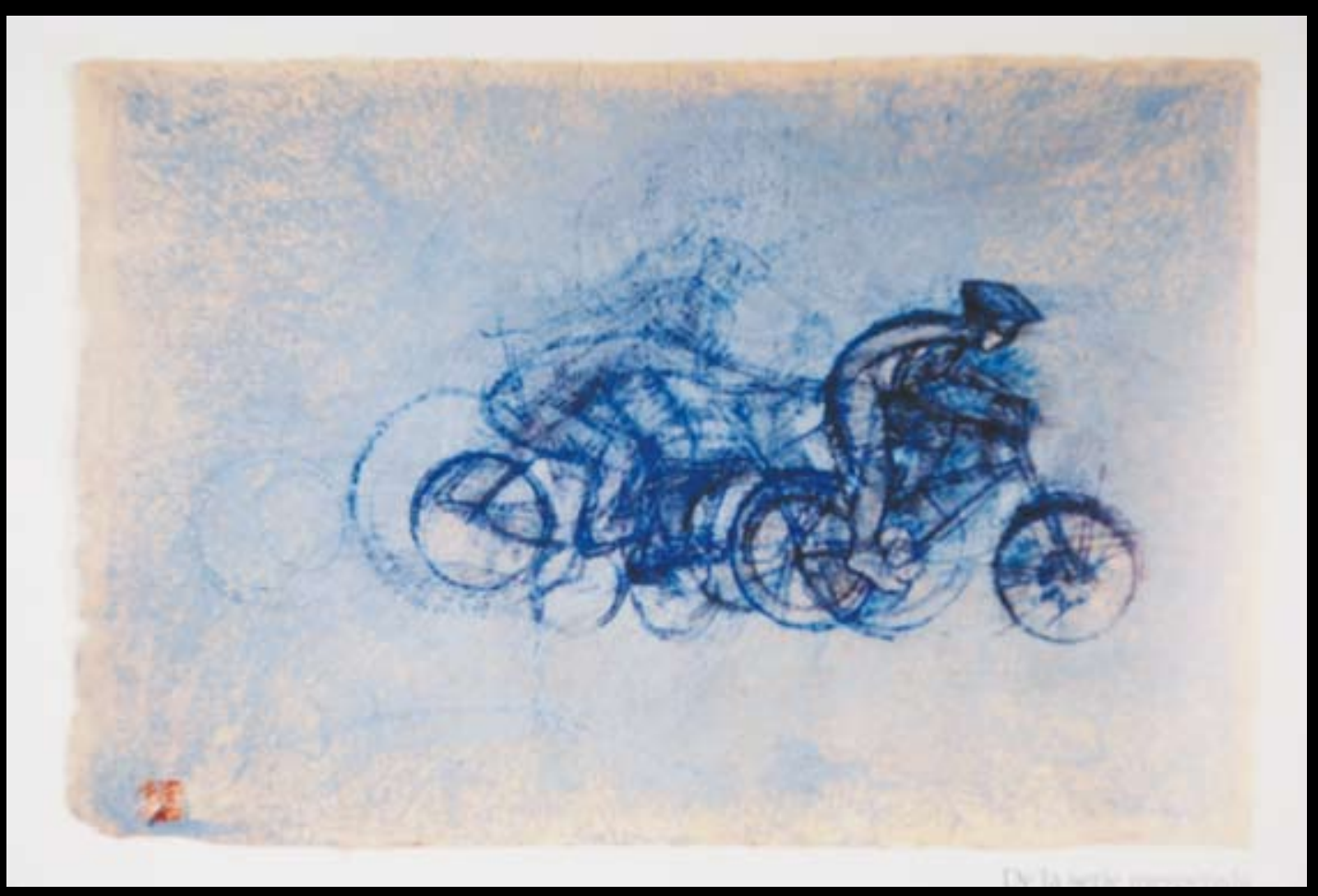

- Las relaciones Europa-América

Latina: no van por buen camino 